\title{
Monaural localization: An analysis of practice effects
}

\author{
ALAN D. MUSICANT and ROBERT A. BUTLER \\ Department of Surgery (Otolaryngology), University of Chicago, Chicago, Illinois 60637
}

\begin{abstract}
Four listeners (experimental group) received one trial a session for 60 sessions on a monaural localization task in which six loudspeakers, positioned $15 \mathrm{deg}$ apart, were placed on the side of the functioning ear. Four additional listeners (control group) received 60 massed trials first and then one trial a session for 60 sessions. The stimulus consisted of a single train of five highfrequency pulses. An ANOVA conducted on the 60 trials distributed over sessions showed that the experimental group's performance was significantly inferior to that of the control group. Members of the former continued to displace sounds toward the open ear throughout the entire test. Those of the control group exhibited this tendency, but to a much lesser extent. The experience of locating 60 massed trials clearly had a robust effect on subsequent performance. We contend that, by virtue of presenting only one trial a session, the magnitude of the massed-trial effect was little attenuated by our tests to measure it. We interpret our substantive finding to mean that during the 60 massed trials, the control group formed a perceptual representation of the auditory space which served it well when placed on the one-trial-a-session regimen. Those in the experimental group were unable to develop an adequate representation of the auditory space, presumably because their monaural localization experience was restricted to only one trial a session.
\end{abstract}

The history of research on auditory space perception has been dominated by the search for and study of cues that govern directional hearing. Interaural time differences, interaural intensity differences, and sound spectrum have all been shown to be fundamental to the localization process (for reviews, see Durlach \& Colburn, 1978; Mills, 1972). Prior auditory experience in the localization task, when researchers have evaluated this variable, has been found to exert a general, positive influence on performance. Within limits, proficiency on the task improves with practice, but since investigators have been intent on studying some other facet of the localization phenomenon, they either have provided a set of practice trials before collecting formal data (e.g., Perrott \& Elfner, 1968 ) or have employed subjects highly experienced as listeners in psychoacoustic research (e.g., Harris \& Sergeant, 1971; Sandel, Teas, Feddersen, \& Jeffress, $1955)$. But the initial exposure to the test environment may exert a profound effect on subsequent performance. Sayers and Cherry (1957), in their list of factors that contribute to auditory space perception, included "knowledge of acoustic properties of 'typical situations' like open doorways, windows, corners of room, etc." Plenge (1974) proposed that when locating a sound source, a listener compares an acoustic signal at any given moment with stored stimulus patterns. He posited both long-term and short-term storages. Of interest is his notion of the latter. When

This research was supported in part by U.S. Public Health Service Grant NS-11154-04. locating a sound in a specific room, one may store knowledge of the room's acoustic idiosyncracies as well as the physical characteristics of the sound source. An implication of Plenge's claim is that each time we enter a different acoustic environment, we must judge the location of various sounds in the context of a special set of acoustic properties that characterize the surrounds. Should this be so, the acquisition of knowledge about the acoustic space must be rapid; we are normally not aware of a disorientation to sounds. Localization research, however, has not addressed this possibility. In the typical experiment, in which listeners are given many trials during a test session, the influence of a brief period of exposure to the room characteristics, if indeed present, is obscured -obscured by the fact that its effect would almost surely be dissipated within the first few stimulus presentations. But this does not mean that the effect would be minimal. If proficient localization performance requires a listener to rapidly integrate the stimulus characteristics peculiar to the sound source with the acoustic properties peculiar to the test room, then we should be able to generate data whose interpretation would be consonant with such a phenomenon. One way would be to limit the opportunity for gaining knowledge about the room acoustics and then determine how well the listener locates sound in that same room. This we attempted in the present study by presenting listeners (experimental group) only one trial a day. The control group was presented with massed trials before participating in the onetrial-a-session regimen. To establish a localization 
task that accentuates the importance of room acoustics, all subjects were tested monaurally - a task in which performance is based on spectral cues.

\section{METHOD}

\section{Subjects}

Eight subjects whose ages ranged from 21 to 29 years participated. All passed the audiometric screening test in which the criterion was set at $10 \mathrm{~dB}$ above audiometric zero for frequencies from 250 through $8,000 \mathrm{~Hz}$ (ISO, Davis \& Kranz, 1964). None had had any prior experience in hearing experiments nor had they ever entered the room in which the experiment was to be carried out.

\section{Apparatus}

Digital logic was controlled through the use of a Grason-Stadler 1200 Modular Programming System. The stimulus, a $3.0-\mathrm{kHz}$ high-pass noise burst, originated from a noise generator (GrasonStadler, Model 1285) and was then tailored by cascading two electronic filters (Spencer-Kennedy Laboratories, Model 302). This arrangement provided a $36-\mathrm{dB} /$ octave rejection slope. The noise signal was gated through an electronic switch (Grason-Stadler, Model 1287B) and then amplified (Crown Amplifier, Model D60). After impedance matching, the signal was delivered to the testroom loudspeakers at $9.0 \mathrm{mV}$.

Six loudspeakers (KLH, 4-in. diam) arranged in an arc at 330 , $315,300,285,270$, and $255 \mathrm{deg}$ azimuth were situated approximately $5 \mathrm{ft}$ from the listener. They were numbered consecutively from 1 through 6 with " 1 " identifying the loudspeaker placed at 330 deg and " 6 " identifying that placed at 255 deg azimuth. The signal delivered to the loudspeakers consisted of one train of five pulses, each $30 \mathrm{msec}$ in duration. Rise-fall time for each pulse was $10 \mathrm{msec}$. Interpulse interval was $300 \mathrm{msec}$. The test room was treated with sound-absorbent material on the walls and ceiling and had carpeting on the floor.
\end{abstract}

\section{Experimental Procedure}

Four subjects were assigned randomly to the experimental group and four were assigned to the control group with the constraint that each group consist of two females and two males. Prior to testing, each subject was rendered monaural by the placement of a Mine Safety Appliance (MSA) ear defender in his/her right ear and then the placement of a MSA muff over the same ear. The procedure has been found to effectively raise the threshold to a broadband noise stimulus in the blocked ear by $28 \mathrm{~dB}$ or more (Belendiuk \& Butler, 1975). To start, thresholds for members of the control group were obtained for sounds emanating from each of the six loudspeakers. Then, at $20 \mathrm{~dB} \mathrm{SL}$, they received 60 localization trials, 10 from each loudspeaker. Order of presentation guaranteed that each loudspeaker would be activated once within consecutive six-trial sets. A different presentation order was constructed for each listener. Intertrial interval was approximately $5 \mathrm{sec}$. Subjects reported via an intercom the number of the loudspeaker they chose as the sound source. (Knowledge of results was withheld.) A headrest and eye-fixation point, positioned at 0 deg azimuth, were provided to minimize head movements. The subjects were told to refrain from moving their heads. From the adjacent room, the experimenter could visually monitor the subjects' actions and was able to determine that they were complying with instructions.

On the day following the 60 massed trials, members of the control group were given one trial a day for 60 sessions. Subjects in the experimental group commenced the study by receiving one trial a day spread out over 60 sessions. Usually, subjects attended 5 sessions a week; sometimes they would miss a day, but rarely were 2 of the 5 sessions per week missed. Irregular weekly attendance, when it occurred, was not peculiar to members of any one group. Again, the six loudspeakers were activated in a planned, but irregular, order, with each delivering the stimulus 10 times during the course of the 60 sessions. The signal level was set at approximately $20 \mathrm{~dB} \mathrm{SL}$, as determined by averaging thresholds for five laboratory personnel whose auditory acuity met the criterion established for the participants of this study. A trial encompassed the following sequence: First, the subject entered the control room (a typical reverberant room), fitted an ear defender in the right ear canal, and then placed a muff over the same ear. Next, (s)he proceeded to the test room and sat in the chair. The experimenter adjusted the headrest. No conversation intervened. The experimenter then retired to the control room, quietly closing the door, and switched the overhead light in the test room momentarily "off," then "on" again. This signaled to the listener that the pulse train would be delivered within 3 to $5 \mathrm{sec}$. After the pulse train presentation, the listener left the test room and reported his/her choice of loudspeaker to the experimenter. We adopted this procedure to minimize the amount of additional auditory experience a listener might receive while in the test room. At the end of 60 one-trial sessions, the experimental group then received a set of 60 massed trials. As was the case for the control group, these were presented at $20 \mathrm{~dB}$ above threshold measured for sounds originating from each loudspeaker.

Localization performances were evaluated in terms of total error score as well as number of correct responses. An error score was defined as the difference in location between the loudspeaker that was activated and that chosen by the subject as the sound source. Thus, if loudspeaker 4 (285 deg azimuth) was activated and the subject responded by choosing either loudspeaker 3 or loudspeaker 5 , an error score of 1 was recorded. Had the choice been either 2 or 6 , an error score of 2 would have been recorded, etc. An error score of 0 was entered when the listener made a correct choice.

\section{RESULTS}

Using error score as an index of localization proficiency, we chose for analysis three components of the data on distributed trials and their respective interactions: (1) differences between experimental and control groups; (2) differences between loudspeaker locations; and (3) differences among consecutive blocks of trials. The loudspeaker locations were divided between those nearer the front $(330,315$, and $300 \mathrm{deg})$ and those nearer the side $(285,270$, and $255 \mathrm{deg}$ ); trials were partitioned in blocks of 12 . Each of the three main effects was statistically significant [groups, $\mathrm{F}(1,6)=15.16, \mathrm{p}<.01$; loudspeaker choice, $F(1,6)=14.02, p<.01$; trial blocks, $F(4,24)=4.56, p<.01]$. One of the interactionsLoudspeaker Location by Groups-was also significant beyond the .01 confidence level $[F(1,6)=18.56$, $\mathrm{p}<.011$. Table 1 provides a detailed view of the data on both distributed and massed trials. Here, apparent locations of the sound source are plotted against their actual locations. The numbers represent frequency of location judgments. Perhaps the most informative aspect of this table is that the significant differences that resulted from the ANOVA arose primarily from the fact that the experimental group showed a strong propensity to displace sounds toward the side $(270 \mathrm{deg})$. As a consequence, the experimental group made larger error scores, which occurred mainly when the sounds originated from the frontalmost loudspeakers. Indeed, statistically significant group differences, loudspeaker location dif- 
Table 1

Apparent Location vs. Actual Location for Massed and Distributed Trials

\begin{tabular}{|c|c|c|c|c|c|c|}
\hline \multirow{2}{*}{$\begin{array}{c}\text { Actual } \\
\text { Location }\end{array}$} & \multicolumn{6}{|c|}{ Apparent Location } \\
\hline & 1 & 2 & 3 & 4 & 5 & 6 \\
\hline \multicolumn{7}{|c|}{ Experimental Group 1: Distributed Trials } \\
\hline 1 & 4 & 18 & 8 & 7 & 2 & 1 \\
\hline 2 & & 8 & 16 & 7 & 4 & 5 \\
\hline 3 & & 1 & 6 & 16 & 11 & 6 \\
\hline 4 & & & 3 & 4 & 24 & 9 \\
\hline 5 & & 1 & 1 & 4 & 24 & 10 \\
\hline 6 & & & & 1 & 21 & 18 \\
\hline
\end{tabular}

Experimental Group 2: Massed Trials

$\begin{array}{rrrrrrr}1 & 8 & 16 & 14 & 2 & & \\ 2 & 2 & 13 & 9 & 12 & 3 & 1 \\ 3 & & 1 & 18 & 13 & 6 & 2 \\ 4 & & & 1 & 13 & 19 & 7 \\ 5 & & & & 2 & 16 & 22 \\ 6 & & & & & 10 & 30\end{array}$

\begin{tabular}{|c|c|c|c|c|c|c|}
\hline \multicolumn{7}{|c|}{ Control Group 1: Massed Trials } \\
\hline 1 & 19 & 15 & 5 & 1 & & \\
\hline 2 & & 15 & 21 & 2 & 1 & 1 \\
\hline 3 & & 4 & 22 & 14 & & \\
\hline 4 & & 1 & 5 & 24 & 9 & 1 \\
\hline 5 & & & 3 & 17 & 14 & 6 \\
\hline 6 & & & 1 & 5 & 11 & 23 \\
\hline \multicolumn{7}{|c|}{ Control Group 2: Distributed Trials } \\
\hline 1 & 25 & 15 & & & & \\
\hline 2 & 3 & 23 & 13 & 1 & & \\
\hline 3 & & 3 & 24 & 12 & 1 & \\
\hline 4 & & & 8 & 23 & 8 & 1 \\
\hline 5 & & & & 12 & 18 & 10 \\
\hline 6 & & & & 2 & 13 & 25 \\
\hline
\end{tabular}

Note-The numbers 1 through 6 represent loudspeakers positioned at 330 through $255 \mathrm{deg}$ azimuth, respectively. The entries represent frequency of loudspeaker choices.

ferences, and Location by Group interaction all stem from the fact that the experimental group, in contrast with the control group, infrequently perceived sounds as emanating from near the front (NewmanKeuls post hoc analysis, $\mathrm{p}<.05$ ).

The basis for the significant differences among consecutive blocks of distributed trials is illustrated in Figure 1b, where we have plotted mean error score per subject separately for the experimental and control groups. Although the experimental group generated a larger error score throughout the one-trial-asession regimen, both groups showed a reduction in error score during the first three 12-trial blocks of the distributed sessions. Pooling the data for both groups and conducting a Newman-Keuls post hoc comparison of treatment revealed that error scores on Block 1 and on Block 2 differed from those on Block 3, Block 4, and Block $5(p<.05)$. Error scores on the last three blocks did not differ from one another $(p>.05)$. Also plotted in Figure 1 are the mean error scores for the massed trials. The sequence of massed and distributed trials that obtained for each group is maintained in the figure. The experimental group, which received the distributed trials first, improved significantly (i.e., smaller error scores) $(t=8.1, p<.01)$ when locating the massed trials. The control group, which received the massed trials first, performed only marginally better when tested on distributed trials $(\mathrm{t}=1.9, \mathrm{p}>.05)$.

While our analysis searched for the differences between the experimental and control groups with emphasis on performance when the sounds were presented once a session, we should point out that the number of correct responses made by each group was significantly above chance expectation according to our calculations of binomial probability $(p<.05)$. In addition, chi-square tests indicated that error score distribution differed significantly from the distribution that would obtain had listeners chosen the loudspeakers at random $(p<.01)$. While the experimental group performed significantly worse on the distributed trials than did the control group, no significant differences between groups in performance were present on the massed trials $(t=.9, p>.05)$. We should mention, however, that the experimental group generated a larger total error score (197) on the 60 massed trials than did the control group (149).

\section{DISCUSSION}

Monaural listeners can locate sound originating in the horizontal plane on the basis of spectral cues provided primarily by the pinna. Distort it and performance deteriorates (Butler, 1975; Gilse \& Roelofs, 1930). But, in order to utilize spectral cues effectively, we suggest that listeners must form an internal representation of the acoustic surrounds, more specifically, a representation of the spatial configuration that describes the relative location of the sound sources. We suggest further that, under everyday listening conditions, this is accomplished rapidly. When entering a new or different space, listeners, both monaural and binaural, perceive sounds generated simultaneously and successively from sundry directions.

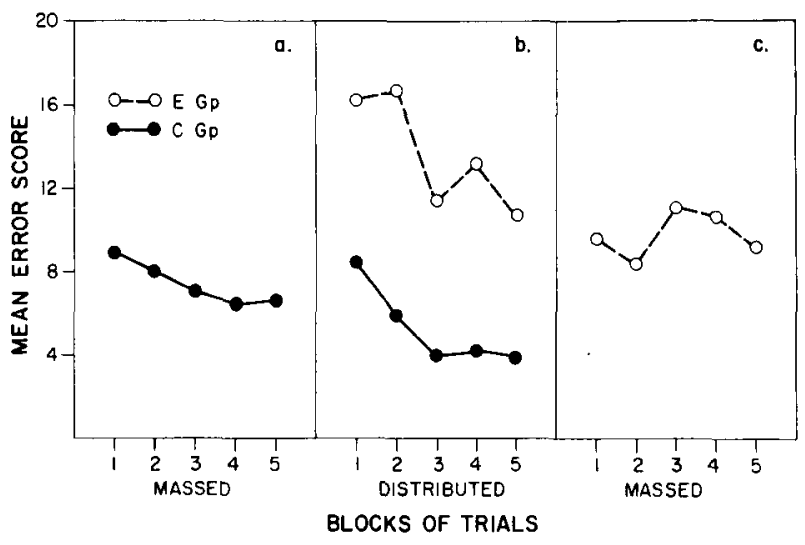

Figure 1. Mean error score per listener as a function of trial blocks. 
The various physical cues for direction are integrated in the acoustic context of the changed environment, and an optimal auditory orientation is achieved. Several aspects of our data are consonant with this idea, but, before discussing them, we should state again how we sequenced our tests. The experimental group received 60 distributed trials, then 60 massed trials; the control group received 60 massed trials, then 60 distributed trials. As we anticipated, subjects who began with 60 massed trials (control group) made lower error scores than those who began with 60 distributed trials (experimental group). We propose that the subjects in the control group performed better because they were given the opportunity to compare the perceptual characteristics of one sound with those presented previously within the same session. As a consequence of this experience, these subjects formed a more adequate internal representation of the auditory space. Furthermore, this internal representation was not degraded when these same subjects were placed on the one-trial-a-session regimen. We base this statement on the finding that performance proficiency during the distributed trials was approximately the same as that recorded for the massed trials. In other words, a cognitive map of the acoustic environment was formed by the end of the 60 massed trials and it remained intact even though listeners no longer had the chance to compare the perceptual characteristics of one sound with those of sounds presented a few seconds earlier. Indeed, the previous trial in the sequence preceded the current trial by anywhere from 24 to $72 \mathrm{~h}$.

To appreciate the importance of massed trials in what we hypothesize to be a formation of an adequate internal representation of the sound field, one need only to consider performance of the experimental group, a group originally deprived of the massed-trial experience. Given only distributed trials on the first 60 sessions, this group performed significantly worse than the control group when the latter was placed on the distributed trials regimen. The main difference in performance between the groups was that the experimental group exhibited a strong propensity to displace the stimulus toward the open ear, that is, away from the midline and nearer $270 \mathrm{deg}$ azimuth. This is not an unusual occurrence for normal-hearing listeners who are suddenly rendered monaural. We attempted to attenuate, if not remove, this judgmental bias from our experiment by placing the most frontal loudspeaker $30 \mathrm{deg}$ off midline and toward the function ear rather than on the midline. We assumed that by doing so, the arc covered by the loudspeakers-330 through $255 \mathrm{deg}$ azimuth-would fall within the monaural auditory field. Yet, the experimental group rarely reported sounds as originating from loudspeaker $1(330 \mathrm{deg})$ (see Table 1). More than likely, a sound from this position was perceived as originating from loud- speaker 2 . Sounds from loudspeaker 2 , in turn, were likely to be perceived as coming from loudspeaker 3 . Those from loudspeakers 3 and 4 were also displaced progressively toward a position directly in line with the open ear, that is, loudspeaker 5. This failure to establish a perceptual anchoring point for the frontal end of the arc, viz., $330 \mathrm{deg}$ azimuth, thus led to the concatenation of errors. With respect to the other end of the arc, sounds emanating from loudspeaker 6 ( $255 \mathrm{deg}$ ) were just as likely to be reported from loudspeaker $5(270 \mathrm{deg})$ as from loudspeaker 6 . The control group, on the other hand, appeared to anchor both ends of the arc relatively firmly, and this may account for the fact that sounds from all loudspeakers were located significantly above chance. In terms of our hypothetical framework, the internal representation of the acoustic space was well articulated. Members of the control group received one other acoustic experience in the test room besides the 60 massed trials. Before the first localization test commenced, thresholds for pulses generated by each loudspeaker were obtained via the method of limits. Whether this event made a difference remains problematical.

Not to be overlooked was the finding that the experimental group still performed above chance level on the 60 distributed trials, as measured in terms of both number of correct responses and distribution of error scores. Hence, members of this group must have utilized the available spectral cues to locate temporally isolated pulse trains. Their utilization, however, was simply less effective than that demonstrated by the control group.

One view of our data is that they merely confirm again the ubiquitous finding that performance improves with practice. But we contend that they have more specific implications: They identify, for the monaural listening situation, what seems ostensibly to be the basis of that improvement associated with practice. Namely, listeners anchor the extreme positions of the array of sound sources, thereby correcting the localization bias exhibited by those temporarily rendered monaural. Proper anchoring according to our thesis was a consequence of the adequate internal representation of the acoustic space, which, in turn, was brought about by the experience of participating in a massed-trial test session. Hence, the data are consistent with the notion that an initial exposure to a sound field is of utmost importance for precise auditory orientation. And lastly, the data uncover what to us was a surprising phenomenon; viz., without knowledge of results, monaural localization of sound can improve when the listener is presented a short train ( 5 pulses) of stimuli only about four or five times a week. It may be that those sounds which inevitably are generated by a person entering the test room and occupying the chair contributed to his knowledge of the room acousticsknowledge that was employed to locate the forth- 
coming train of pulses. Should this be so, it further strengthens our thesis that we rapidly and continually integrate all components of the sound field when making localization judgments.

\section{REFERENCES}

BetevDilk, K., \& Butler, R. A. Monaural localization of lowpass noise bands in the horizontal plane. Journal of the Acoustical Society of America, 1975, 58, 701-705.

Butt.F. R. A. The influence of the external and middle ear on auditory discriminations. In W. D. Keidel \& W. D. Neff (Eds.), tuditory sistem (Vol. 5: Pt. 2) Handbook of sensory physiology. Berlin: Springer-Verlag, 1975.

Divis, H., \& Kranz, F. W. The international standard reference zero for pure-tone audiometers and its relation to the evaluation of impairment of hearing. Journal of Speech and Hearing Research. 1964. 7, 7-16.

Dirl.ach, N. I., \& Colburn, H. S. Binaural phenomena. In F. C. Carterette \& M. P. Friedman (Eds.), Hearing (Vol. 4) Handhook of perception. New York: Academic Press, 1978.
Gısse, V., \& Rok lofs, O. Untersuchungen über die Schallokalisation. Acta Otolaryngologica, 1930, 14, 1-20.

Harris, J. D., \& Sergeant, R. L. Monaural/binaural minimum audible angles for a moving sound source. Journal of Speech and Hearing Research, 1971, 14, 618-629.

Mitss, A. W. Auditory localization. In J. V. Tobias (Ed.), Foundations of modern auditory theory (Vol. 2). New York: Academic Press, 1972.

Perrott, D. R., \& Elfner, L. F. Monaural localization. Journal of Auditory Research, 1968, 8, 185-193.

Plenge, F. On the differences between localization and lateralization. Journal of the Acoustical Society of America, 1974, 56, 944-951.

Sandel., T. T., Teas, D. C., Feddensen, W. E., \& Jeffress, L. A. Localization of sound from single and paired sources. Journal of the Acoustical Society of America, 1955, 27, 842-852.

Sayers, B. M., \& Cherry, E. C. Mechanism of binaural fusion in the hearing of speech. Journal of the Acoustical Society of America, 1957, 29, 973-987.

(Received for publication January 2, 1980; revision accepted May 15, 1980.) 